\title{
The assessment of the tensile with torsion loading interaction using the selected hypotheses and the experiment
}

\author{
Vladimír Chmelko ${ }^{1}$, Pavol Novotný ${ }^{2}$ \\ ${ }^{1}$ Slovak University of Technology, Námestie slobody 17, 81231 Bratislava, Slovakia \\ ${ }^{2}$ Slovnaft, a.s., Vlčie Hrdlo 1, 82412 Bratislava
}

\begin{abstract}
For two steels with different material properties (both cyclic and static ones) were experimentally obtained dependencies $\sigma_{a}=f\left(N_{f}\right)$ and $\tau_{a}=f\left(N_{f}\right)$. The specimens of these materials were loaded with multiple tensile and torsion stress combinations. It was found out that the timedepending relationship between the individual stresses was proportional. The results for steel with high ration of $\mathrm{Rm} / \mathrm{Re}$ are different comparing to the results of the second material. In this contribution, there will be confronted the experimentally obtained fatigue life-time magnitudes with selected multiaxial fatigue life-time hypotheses like Findley, McDiarmid, Dang-Van, Carpinteri-Spagnioli, and Margetin-Ďurka-Chmelko.
\end{abstract}

\section{Introduction}

The large number of cumulation hypotheses of fatigue damage which take into account the multi-axial stress state does not need to be further expanded. Rather, the generalization of existing approaches (if at all possible) into a unified fatigue life assessment methodology for multi-axial stress would be a better approach. In this paper, one of the ways of thinking which could be followed for solving the problem is outlined.

Two steels selected for analysis are common structural steels often used in engineering practice. Their difference is in a different $\mathrm{Rm} / \mathrm{Re}$ ratio. For C55 steel, the strength to yield strength ratio is 2.5 , for St-52 steel is less than 1.1. Fatigue life curves for normal stress (alternating tensile tests) and shear stress (alternating torsion tests) were obtained for both steels. This was followed by cyclic loading of specimens of each of the steels with tensile and torsion combinations to involve a lifetime region of order $10^{4}$ to $10^{6}$. The stress criteria Findley, McDiarmid, Dang-Van, Carpinteri-Spagnioli and Margetin-Ďurka-Chmelko were chosen for the fatigue life calculation. Computational results are confronted with experimental obtained lifetimes.

1 Corresponding author: vladimir.chmelko@stuba.sk 


\section{Properties of analysed steels}

Materials ST-52 and C55 are medium strength non-alloy steels with distinctly different strengthening modules. All material properties were obtained by direct measurement. The shape of the test specimens for all tests was chosen so as not to cause any factors affecting the results (e.g., the samples did not have an internal opening).

Tensile diagrams of both steels documenting their differences are shown in Fig.1.
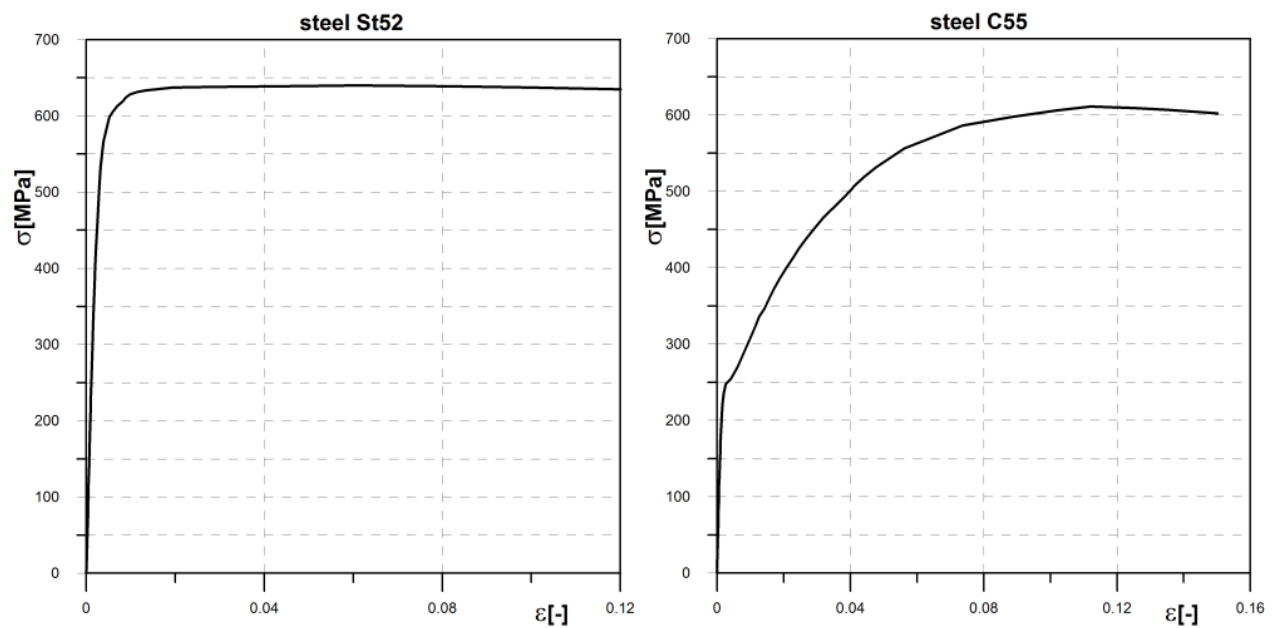

Fig. 1. Tensile diagram for analysed steels St52 and C55

Regression curves of experimentally obtained dependencies $\sigma_{a}=f\left(N_{f}\right)$ and $\tau_{a}=f\left(N_{f}\right)$ for both steels are in Fig.2 and Fig.3. The cycles labeled as $N_{f}$ represent fatigue life up to fracture, as well as the following tensile-torsion combined tests.
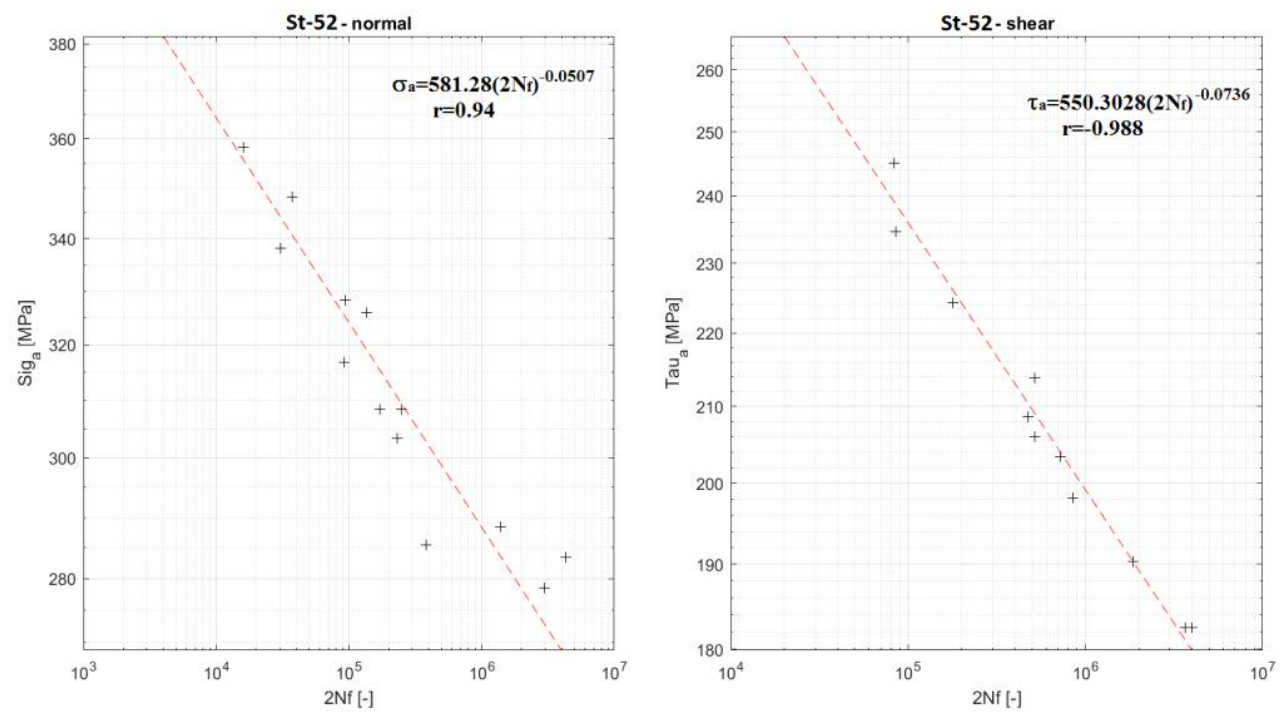

Fig. 2. Basquin curves for analysed steel St52 

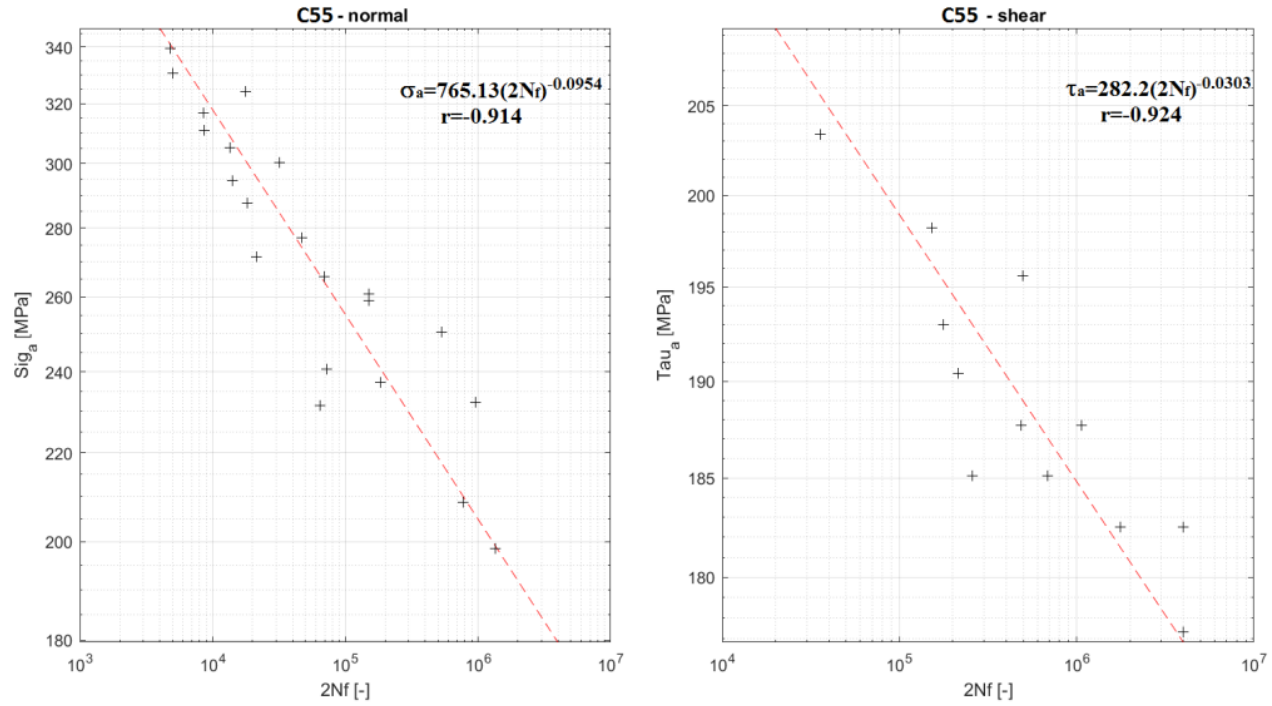

Fig. 3. Basquin curves for analysed steel C55.

\section{Obtained results}

The specimens of these materials were loaded with multiple tensile and torsion stress combinations in frame of electrohydraulic pulsator MTS 370.02. It was found out that the time-depending relationship between the individual stresses was proportional. Frequency of cycling was less than $5 \mathrm{~Hz}$. Obtained experimental values of life-time for both steels was confronted with selected multi-axial fatigue damage criterions as shown in the diagrams Fig.4-Fig.8. The prediction intervals for $90 \%$ probability of occurrence of experimental points are shown in diagrams by dashed lines.
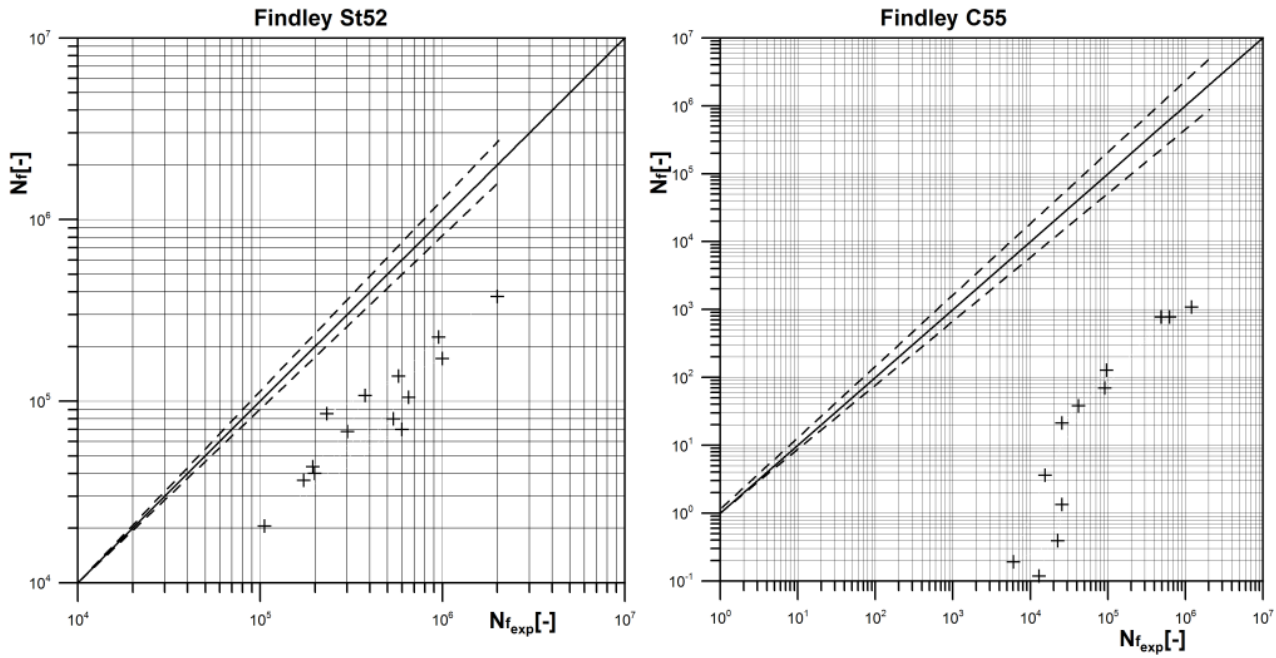

Fig. 4. Confronting of lifetime applying Findley approach [1] and experimental results for analysed steels 

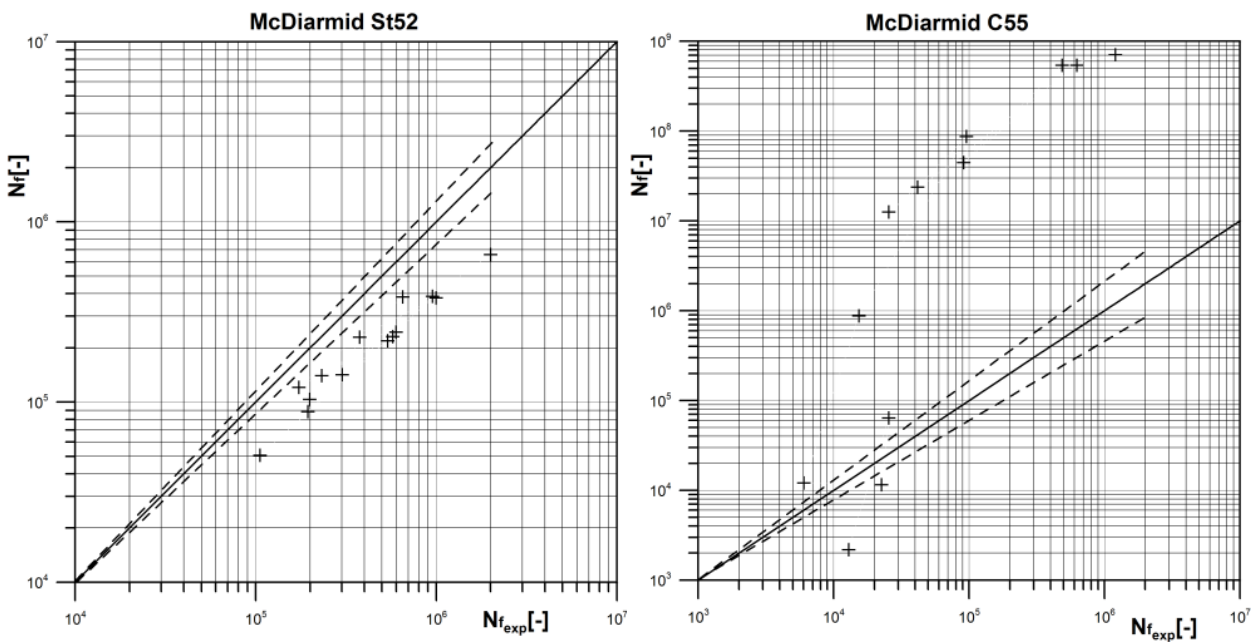

Fig. 5. Confronting of lifetime applying McDiarmid approach [2] and experimental results for analysed steels
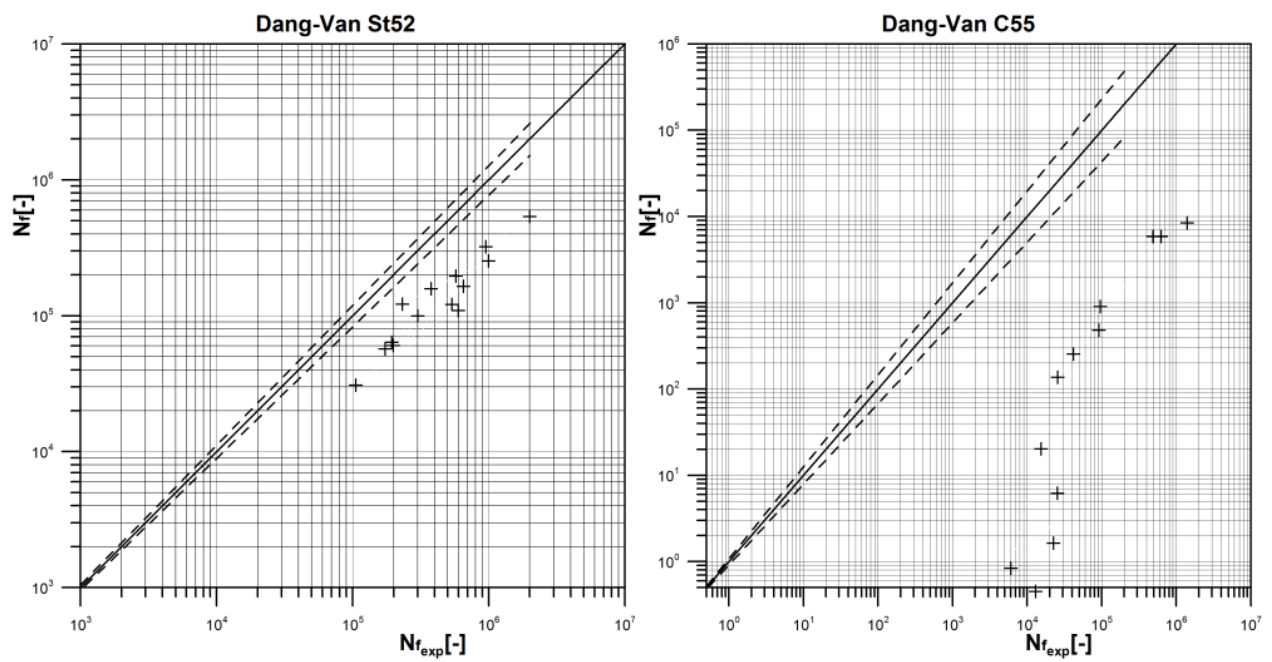

Fig. 6. Confronting of lifetime applying Dang-Van approach [3] and experimental results for analysed steels 

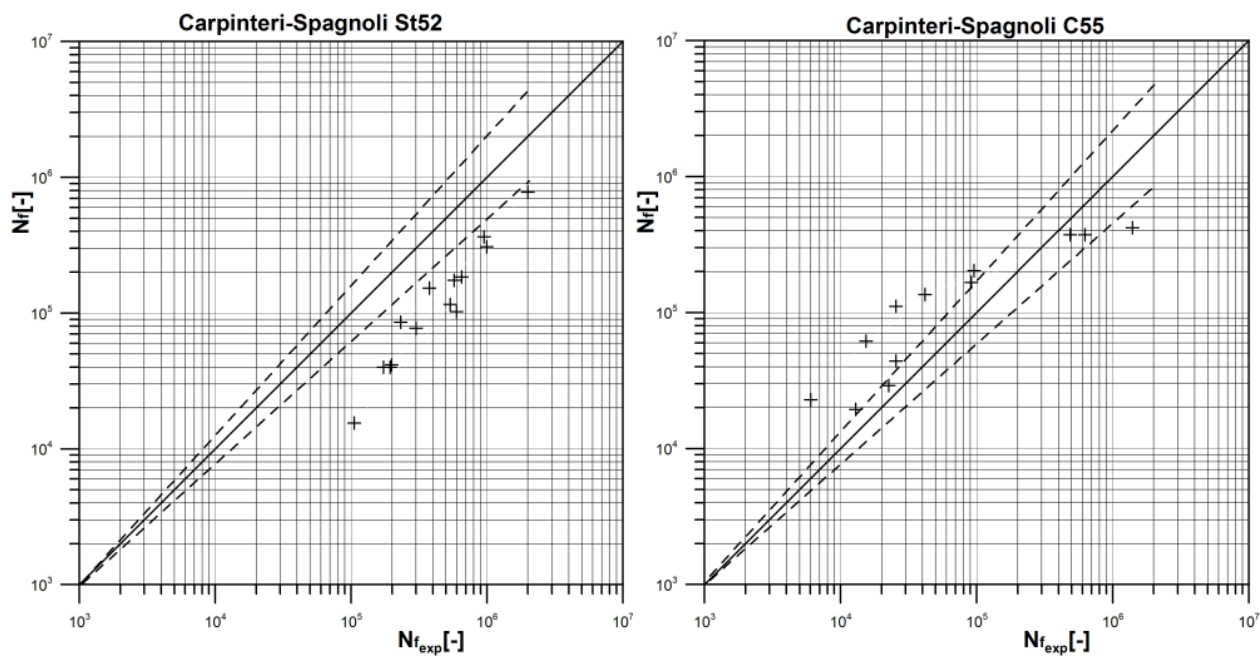

Fig. 7. Confronting of lifetime applying Carpinteri-Spagnoli approach [4] and experimental results for analysed steels
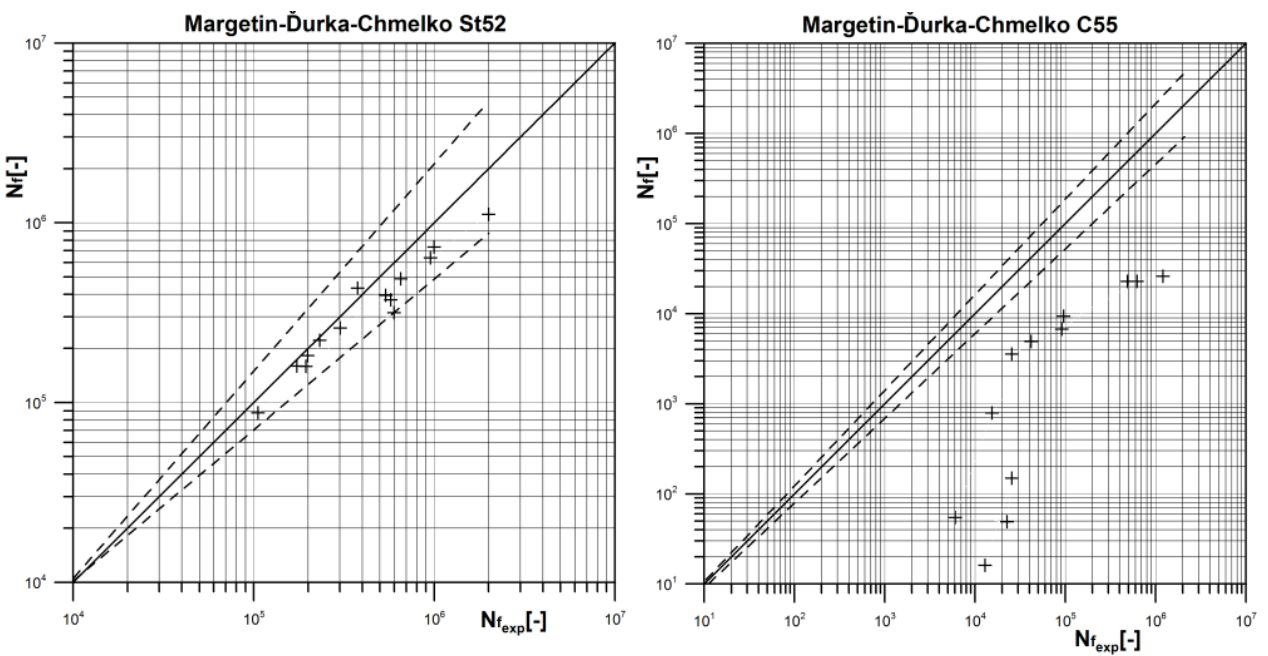

Fig. 8. Confronting of lifetime applying Margetin-Ďurka approach $[5,6]$ and experimental results for analysed steels

\section{Discussion of results and conclusions}

In this paper, there were confronted the experimentally obtained fatigue life-time values with calculated values using selected multiaxial fatigue life-time hypotheses like Findley, McDiarmid, Dang-Van, Carpinteri-Spagnioli, and Margetin-Durka-Chmelko (MDC). The results of the fatigue tests (presented with statistical intervals of probability) and the results obtained by the computational approaches are very different for the steels analysed.

For St52 steel

- all the used approaches resulted in conservative results

- the MDC approach best matched the experimental results

- the most conservative results were obtained by Findley's approach 
- the results of using the McDiarmid, Dang-Van and Carpinteri-Spagnoli approaches are very similar

For C55 steel

- the Findley and Dang-Van approaches have led to very conservative results

- the MDC approach has led to less conservative results, but correlation with experimental results is also unsatisfactory

- the McDiarmid and Carpinteri-Spagnoli approach has in majority led to nonconservative results, while Carpineri-Spagnoli's approach has the best correlation with experimental results

Overall, the results of computational approaches are generally poorly satisfactory for both steels, and even very unsatisfactory for C55 steel. The MDC criterion has a real best correlation with experimental results, but it does not capture the reality for C55 steel.

The specificity of C55 steel can capture some of the deformation criteria [7], which often require additional material parameters. The results of material C55 lead to the idea of introducing an additional material parameter that would correct such specifics using relatively simple stress criteria.

This work was supported by the Slovak Research and Development Agency under the contract No. APVV-17-0666 and by the Research \& Development Operational Programme funded by the ERDF ITMS: 26240220084 Science city Bratislava.

\section{References}

1. Findley, W. N., Fatigue of metals under combinations of stresses, Trans ASME, 79, 1337-1338, (1957)

2. McDiarmid, D. L., A general criterion for high cycle multiaxial fatigue failure, Fatigue Fract Eng Mater Struct 14, 429-453, (1991)

3. Dang-Van, K., "Macro-Micro Approach in High-Cycle Multiaxial Fatigue" in Advances in Multiaxial Fatigue., ASTM STP 1191, D.L. McDowell and R. Ellis, Eds., American Society for Testing and Materials, Philadelphia, 120-130, (1993)

4. Carpinteri, Spagnoli, A., Vantadori, A.S., A multiaxial fatigue criterion for random loading, Fatigue of Engineering Materials and structures 26, 515-522, (2003)

5. M. Margetin, R. Durka, V. Chmelko, Multiaxial fatigue criterion based on parameters rom torsion and axial S-N curve, Fattura ed Integrita Strutturale, 10, 146-152, (2016)

6. M. Margetin, M. Šulko, Fatigue lifetime estimation of bearing pin of console manipulator loaded with multiaxial random loading. Procedia Engineering, 213, 788796, (2018)

7. D.F. Socie, G.B. Marquis, Multiaxial fatigue. Society of Automotive Engineers, Inc. Warrendale, Pa., (2000) 